\title{
Raising Cultural Awareness through Implicit Exposure to Culturally-loaded Vocabulary
}

\author{
Mohammad Ali Heidari-Shahreza (Corresponding author) \\ Department of English, Faculty of Humanities, Shahreza Branch, Islamic Azad University, \\ Shahreza, Iran \\ Tel: 98-31-5329-2008Ｅ-mail: maheidari.sh@gmail.com
}

Received: June 17, 2014 Accepted: June 23, 2014 Published: August 12, 2014

doi:10.5296/ijch.v1i2.5824 URL: http://dx.doi.org/10.5296/ijch.v1i2.5824

\begin{abstract}
The study explored the possible effects of exposure frequency on implicit acquisition of culturally-loaded words. In the context of the study, culturally-loaded words were defined as those L2 words which had significantly-different cultural connotations with respect to learners' first language (and culture). Within a quasi-experimental research design, 75 adult EFL learners took part in the study. Their gains in three culture-related aspects of lexical knowledge, through implicit exposure to target words, were measured. The findings confirmed that exposure frequency had a positive effect on vocabulary knowledge as a continuum of one to seven encounters could tell. In addition, it was revealed that receptive knowledge of meaning and form (RMF) as well as receptive knowledge of associations (RA) responded faster to implicit exposure than productive knowledge of associations (PA). Furthermore, whereas gains in vocabulary knowledge for RMF and RA were significant even after three encounters, for this latter subknowledge, at least seven encounters were needed to reach significant results.
\end{abstract}

Keywords: Implicit exposure, Exposure frequency, Culturally-loaded words, EFL learners

\section{Introduction}

\subsection{Vocabulary Knowledge}

Vocabulary knowledge (or lexical knowledge) plays a significant role in both first language (L1) and second language (L2) acquisition. Research shows children spend their first years of L1 acquisition on mainly mastering different aspects of lexical knowledge (Brent \& Siskind, 
2001; Meara, 2012; Rott, 2007). Similarly, vocabulary knowledge (and competence) is regarded as a core component of L2 proficiency and is an important indicator of command on receptive (i.e. reading and listening) and productive (e.g. writing and speaking) language skills (Brown, Waring \& Donkaewbua, 2008; Kim, 2011; Nation, 2013).

Research on vocabulary knowledge in general and the acquisition, development and retention of L2 (or foreign) vocabulary in particular, however, has come to the fore recently (see for example, Broady, 2008; Meara, 2012). Following this line of research, scholars have been investigating quite a number of issues especially what vocabulary knowledge entails. In simpler terms, what is meant by knowing a word? Some scholars believe that lexical knowledge entails a command on a set of interrelated subknowledges such as knowledge of meaning and form, grammatical or morphological knowledge which in all amount to the construct of vocabulary (e.g. Nation, 1990, 2001; Qian \& Schedl, 2004; Webb, 2007, 2013). Some others view vocabulary knowledge as accumulating phases of mastery based on which learners begin language learning with an elementary familiarity with most common words of a language and (probably) progress to the highest levels of mastery where they can readily use L2 vocabulary in communicatively-appropriate situations such as delivering a speech or having a pep talk with a friend (Brown, 2011). Recently, vocabulary knowledge is increasingly viewed as a multidimensional psychological trait which is in sharp contrast with the traditional view on lexical knowledge as a single dimension. That is to say, there is a growing tendency among researchers that knowing a word requires mastery on various aspects of formal and semantic knowledge (see also Hellman, 2011). For example, Nation (2001) believes that vocabulary knowledge incorporates three macro levels of form, meaning and use, which each consists of a number of micro dimensions to cover receptive and productive aspects of knowing words. Therefore, in general, it can be said that lexical knowledge as a multidimensional set of interrelated subknowledges entails knowing primary meaning(s) of a word, its appropriate uses in authentic contexts, its grammatical and morphological properties and the associations a word has with other L2 words (Brown, 2011).

\subsection{Implicit Exposure to L2 Vocabulary}

There are implicit (incidental) and explicit (intentional) approaches to acquire and develop L2 vocabulary. Implicit vocabulary acquisition which is also interchangeably called incidental vocabulary acquisition refers to learning new words while reading or listening to L2 (Heidari-Shahreza \& Tavakoli, 2012). That is, learners acquire new vocabulary while their focus of attention is on comprehending the meaning of the written or spoken text. This approach is in contrast with an explicit (or intentional) approach to L2 vocabulary acquisition which centers around learning L2 new words through explicit instruction in language classrooms and devising form-focused tasks (Schmitt, 2008).

There are ongoing debates on the extent either approach is efficient or how (and when) they should be employed (Eckerth \& Tavakoli, 2012). Nevertheless, there is a general consensus that implicit exposure is a notable means of L2 vocabulary acquisition and development particularly in intermediate and advanced levels of language proficiency, when the first few 
thousand words of a language have already been acquired (Chen \& Truscott, 2010; Eckerth \& Tavakoli, 2012; Hairrell, Rupley \& Simmons, 2011; Webb, 2007). Reading passages in L2 or listening to recordings provides learners with the opportunity to pick up and retain various characteristics of L2 vocabulary especially those communicative or contextual nuances which otherwise are so hard to master (Heidari-Shahreza \& Tavakoli, 2012).

\section{Research on Implicit Exposure}

Although there are more than three decades of research on implicit vocabulary acquisition and a notable number of studies have explored many aspects of vocabulary acquisition (see Broady, 2008 for a review), there still remain some areas of concern which await further research (Meara, 2012). Hence, the present study probes exposure frequency or number of repetition within the realm of implicit vocabulary acquisition. In the context of this study, exposure frequency refers to how many times learners encounter L2 vocabulary (i.e. English) while they read and comprehend some reading passages (Heidari-Shahreza, Moinzadeh, \& Barati, 2014a). In the next section, a brief account of the studies which are most germane to the scope of the study together with their weak and strong points are mentioned to highlight the gap and pave the ground for the present study.

\subsection{Possible Effects of Exposure Frequency}

One of the pioneering studies on the possible effects of exposure frequency (or number of repetition) was Saragi, Nation and Meister's study (1978). In this study, the participants read the English novel, 'A clockwork orange', in which some Russian words (as target L2 vocabulary) were inserted. The researchers found a significant chance of acquiring new words implicitly (75\% to be exact). The correlation between exposure frequency and acquisition was also determined as 0.34 . Following the same line of research, Horst, Cobb and Meara (1998) using graded readers as a more authentic input, found a correlation of 0.49 . They indicated an index of eight or more encounters for significant gains in vocabulary knowledge. Despite an improved methodology over previous studies, their observation was affected by the context of the target words (TWs, hereafter) especially whether pictures accompanied them or not.

Rott (1999) also embarked on examining the same topic using 95 German learners and 12 English TWs. While she observed that even two encounters could produce significant gains, Hulstijn, Hollander and Greidanus' study (1996) in a similar study did not. Likewise, Waring and Takaki (2003), Pigada and Schmit (2006), and Brown, Waring and Donkaewbua (2008) investigated the possible effects of exposure frequency within the perspective of implicit vocabulary acquisition. In general, these researchers mainly explored the acquisition (and retention) of new L2 vocabulary through reading passages with a focus on their form, meaning and recall. Among other things, they reported that an increased number of encounters to TWs could most significantly affect knowledge of form, meaning recognition and meaning recall within a continuum of 0-20 frequency. Although these studies employed a larger repertoire of TWs and took account of vocabulary acquisition and retention both immediately and after some time, they were limited in that tests of form and meaning recognition (or recall) were mainly used which could not represent and assess the totality of 
vocabulary knowledge as introduced in section 1.1.

Webb (2007), Chen and Truscott (2010) and Heidari-Shahreza and Tavakoli (2012) also investigated the same issue. Their measurement tools, however, were much improved. Initiated by Web (2007), they used a vocabulary post-test which included at least seven subtests measuring various aspects of receptive and productive knowledge of vocabulary such as orthography, parts of speech, meaning and associations. Webb (2007) reported that significant gains could be observed after ten encounters to TWs. Chen and Truscott (2010), among other things, found that exposure frequency could affect receptive subknowledges such as receptive knowledge of orthography or parts of speech more than their productive counterparts immediately. Heidari-Shahreza and Tavakoli (2012) concluded that increasing the amount of exposure had the highest effect on semantic aspects of vocabulary knowledge such as meaning and associations. These aspects, however, were more difficult to retain by the participants after several weeks.

These studies, however, were limited in a number of ways. Web's study (2007) employed invented words in isolated sentences. That is, the TWs were not real words. In addition, they were used out of context. Hence, its ecological validity and authenticity were not optimally ensured. Chen and Truscott's study (2010) was limited in that the contextual position of the TWs was not fully controlled. Therefore, the findings could also be affected by how informative the contexts were. In Heidari-Shahreza and Tavakoli's study only 10 TWs were used. In addition, the cultural connotations of the TWs could affect the results beyond the scope of the study.

Recently, Heidari-Shahreza, Moinzadeh and Barati (2014a) explored possible effects of implicit exposure to L2 vocabulary, using a much-improved methodology and a larger sample of TWs. The findings were much in line with those of Heidari-Shahreza and Tavakoli's (2012). This study, however, focused on four various sets of vocabulary. Thus, the observed findings could be affected by this factor.

In sum, while there have been a number of studies on exposure frequency, there is not yet clear how different aspects of vocabulary knowledge are affected by increasing the number of encounters to TWs. Moreover, the relevant literature does not yet indicate any optimal number of repetitions.

\subsection{Acquisition of Culturally-loaded Words}

In addition to need for further research on exposure frequency (as mentioned above), the acquisition and retention of cultural aspects of vocabulary knowledge remain unquestioned. In this regard, 'cultural loadedness' is of primary importance. It refers to significantly-different cultural connotations some L2 vocabulary may convey (Heidari-Shahreza, Moinzadeh, Barati, 2014 b). These connotations (or secondary meanings) are most often connected to negative feelings, communicative norms and ethical values (Liu \& Zhong, 1999). For instance, the English word 'date' meaning a romantic appointment or 'girlfriend' may be properly regarded as a 'culturally-loaded' word for Iranian EFL learners because within the Islamic context of Iran, such words refer to relations among male and 


\section{Macrothink}

females which are not authorized religiously and to some extent socially. The notion of cultural loadedness is not, however, confined to negative concepts. It may also refer to schematically-different social acts. As an example, the word 'wedding' refers to a pleasant social event which is highly-valued in different cultures. Nevertheless, this word is regarded as a culturally-loaded word because the way a wedding is celebrated significantly varies across Iranian and Western culture. Therefore, this word triggers quite a different set of schemas (i.e. mental images of social acts) for Iranians in comparison with, say, Americans.

Although culturally-loaded words have always attracted some attention (see Lado, 1972), they have not yet been investigated from the viewpoint of implicit vocabulary acquisition in general and the effects of exposure frequency in particular (Heidari-Shahreza, Moinzadeh, \& Barati, 2014b). To the best of the researcher's knowledge, the only exception is Heidari-Shahreza, Moinzadeh, \& Barati's study (2014 b) in which they took a brief account of the acquisition and retention of culturally-loaded and culturally-neutral words. This study, however, was mainly concerned with comparison between lexicalized and culturally-loaded words not the effects of exposure frequency on culturally-loaded words.

Therefore, the present study aimed at investigating the effects of exposure frequency on the acquisition of culturally-loaded words as an area of research which (as indicated in sections 2.1 and 2.2.) needed further research.

\section{Methods}

The present study employed a quasi-experimental design and a number of quantitative statistical procedures to explore the topic under investigation. The independent variable was exposure frequency or the number of encounters to the TWs. The dependent variable was different aspects of vocabulary knowledge.

The following research questions guided the study:

1) What are the possible effects of implicit exposure to TWs on the acquisition of vocabulary knowledge?

2) Do the observed effects, if any, vary across different aspects of vocabulary knowledge?

\subsection{Participants}

The population was young adult EFL learners. Through a convenience sampling, 75 learners in three equal experimental groups participated in the study. They were selected from two universities in Iran. The average age of the participants was 21 . To ensure the homogeneity of the participants in terms of their general language proficiency, their English score in National University Entrance Examination and Oxford Solutions Placement Test were taken into account. Furthermore, the Vocabulary Levels Test (Nation, 1990) was conducted to make sure the participants had the same vocabulary knowledge at the time of the treatment (see Table $1)$. 
Table1. Participants of the study

\begin{tabular}{|c|c|c|c|c|c|}
\hline Group & No & Age & Score on University Test & Score on Placement Test & Score on VLT \\
\hline G1 & 25 & 21 & $43 \%$ & 56 out of 70 & 26 out of 30 \\
\hline G3 & 25 & 21 & $49 \%$ & 53 out of 70 & 25 out of 30 \\
\hline G7 & 25 & 21 & $45 \%$ & 54 out of 70 & 27.5 out of 30 \\
\hline
\end{tabular}

\subsection{Materials \& Instruments}

In this section, the materials and instruments used at different stages of the study are briefly described.

\subsubsection{Target Words (TWs)}

This study made use of 20 TWs (see Appendix One). The TWs were selected from the corpus of culturally-loaded words collected by Heidari-Shahreza, Moinzadeh and Barati (2014b). The rationale behind this selection was that the present study focused on the same TWs and participants. Moreover, the TWs in both studies were based on the same research design and within a similar research scope (see Heidari-Shahreza, Moinzadeh, \& Barati (2014b) for further information). The TWs included eight verbs, eight nouns and four adjectives because these lexical categories were among the most common bulk of L2 vocabulary. In addition, following Heidari-Shahreza, Moinzadeh and Barati (2014 a, b), their frequency of occurrence, level of difficulty and their conceptual familiarity were kept under control.

\subsubsection{Texts}

Based on the research design and the intended number of encounters to the TWs, 13 reading passages were employed. Seven of these contained the TWs. Hence, they were called main (M) passages. The other six passages did not contain the TWs. Thus, they only served as distracter (D) passages to balance the amount of written input each experimental group received. Following Heidari-Shahreza, Moinzadeh and Barati (2014 a, b), the distracters were chosen from a reading book at intermediate level by Kirn and Hartmann (2002). It is also worth mentioning that attempt was made to keep other features of the texts under control. For example all the texts had almost the same length (about 250 words). Moreover, the main and distracter passages were arranged in a way that all groups of participants finished their reading phase with a main passage (see Appendix Two). This way, the research was able to control for the effect of exposure timing.

\subsubsection{Vocabulary Test}

The vocabulary test was a modified version of Web's test of vocabulary knowledge (2007). In the present study, only semantic subtests of the original test were used because the study focused on culturally-loaded words which were semantically-different. In addition, the relevant literature suggested that the semantic areas of vocabulary knowledge such as meaning and associations could be the main venue of lexical differences (see Heidari-Shahreza \& Tavakoli, 2012; Heidari-Shahreza, Moinzadeh, \& Barati, 2014b). Therefore, the vocabulary test used in this study measured three aspects of vocabulary 
knowledge as follows:

Subtest 1. Receptive Knowledge of Meaning and Form (RMF)

To measure this aspect of lexical knowledge, the participants were instructed to translate the TWs into their L1 (i.e. Persian). In doing so, however, they had to provide all necessary features of the TWs. Therefore, in translating the TW, 'pet' for instance, both concepts of 'animal' and 'domestic' needed to be manifested in the participants' translation.

Subtest 2. Receptive Knowledge of Associations (RA)

The participants in this section of the test were required to identify the option which was pragmatically associated with the given TW. Attempt was made to include a concept among the options which bore the main difference between the two cultures at issue. As an example, for the TW 'pray', the location of praying (mosque vs. church) was in focus.

Subtest 3. Productive Knowledge of Associations (PA)

This test was actually the productive version of the previous test. Therefore, instead of identifying the pragmatically-associated word among the options, the participants were asked to provide such a word. For the TW 'date', 'romantic' was, for example, an acceptable answer.

\subsection{Piloting}

Prior to the main study, the above instruments and materials, particularly the three subtests of the vocabulary test were piloted through the same research design with a group of $30 \mathrm{EFL}$ learners who had the same characteristics as the final participants. The researcher had also subsequent interviews with 10 randomly-selected participants of the pilot study to make inquiries about the instruments and materials from the learners' point of view. Through the process of piloting and subsequent interviews, the instructions for the subtests were made clearer and an example was provided for all the subtests. In addition, the reliability coefficient of each subtest was calculated as sufficiently high (see Table 2). Furthermore, it was assured that the participants could properly finish reading passages in 50 minutes. The appropriate timing for different subtests of the vocabulary test was also determined (see Table 3)

Table 2. Cronbach's alpha coefficients of vocabulary subtests

\begin{tabular}{|c|l|c|}
\hline No. & \multicolumn{1}{|c|}{ Vocabulary subtest } & Reliability \\
\hline 1 & Receptive Knowledge of Meaning and Form (RMF) & 0.79 \\
\hline 2 & Productive Knowledge of Associations (PA) & 0.78 \\
\hline 3 & Receptive Knowledge of Associations (RA) & 0.80 \\
\hline
\end{tabular}

\subsection{Procedure}

In the first phase of the study, the participants read seven texts. Based on the research design, however, G1 read only one main and six distracter passages. The other two groups, that is, G3 and G7, read three and seven main passages respectively. Therefore, while G1 had one incidental encounter to the TWs, G3 had three encounters and the learners in G7 received the 
highest frequency of input (i.e. 7 encounters). As mentioned in section 3.3., the reading phase took about 50 minutes. Thus, on average, the participants had almost seven minutes to read each text.

As the second phase, the participants sat for the vocabulary test. They did not expect such a test. Yet, they were told that there would be a test. Doing so, the research made sure the participants were attentive enough during the first phase. The whole test took about 50 minutes. Based on the piloting procedure, the time allotted to different sections of the test was determined to be sufficient (see Table 3).

Table 3. Time limits of vocabulary subtests

\begin{tabular}{|c|l|c|}
\hline No. & \multicolumn{1}{|c|}{ Vocabulary subtest } & Time (min) \\
\hline 1 & Receptive Knowledge of Meaning and Form (RMF) & 15 \\
\hline 2 & Productive Knowledge of Associations (PA) & 15 \\
\hline 3 & Receptive Knowledge of Associations (RA) & 20 \\
\hline
\end{tabular}

\subsection{Scoring Procedure}

Through different phases of the study, the administered vocabulary test was scored systematically by the researcher and two assistants who were experienced EFL teachers with sufficient knowledge in teaching and testing. Before scoring the tests, several meetings were held between the researcher and his assistants to define and clarify the research scope and the scoring procedure. Therefore, all scorers followed the same blueprint in scoring the tests. The inter-rater reliability was also sufficiently high $(0.85)$. To score the vocabulary test, each question item (in each subtest) was assigned half a point. Therefore, each subtest had 10 points and the overall test had 30 points.

\subsection{Data Analysis}

To analyze the scores obtained from the vocabulary test, ANOVA and its non-parametric version Kruskal-Wallis was used. Post hoc Tukey and least significance difference (LSD) tests were also employed to check for any significant relationships between the variables (at $p=.05)$.

\section{Findings}

\subsection{Effects of Implicit Exposure}

Using statistical procedures, mean scores (M) and standard deviations (SD) for groups G1, G3 and G7 were calculated. As Table 4 shows, as the number of encounters to the TWs (i.e. exposure frequency) increases, participants' mean scores rise. In other words, the learners in G7 had a better performance than G3. G3 also outperformed the participants in G1. 
Table 4. Mean scores and standard deviations of vocabulary test

\begin{tabular}{|c|c|c|c|c|c|c|}
\hline Group & \multicolumn{2}{|c|}{ G1 } & \multicolumn{2}{c|}{ G3 } & \multicolumn{2}{c|}{ G7 } \\
\hline Subtest & $M$ & $S D$ & $M$ & $S D$ & $M$ & $S D$ \\
\hline RMF & 2.12 & 0.69 & 4.78 & 2.31 & 8.24 & 5.01 \\
\hline RA & 3.56 & 2.22 & 5.98 & 2.86 & 7.79 & 4.06 \\
PA & 1.45 & 0.53 & 2.32 & 1.40 & 4.02 & 3.33 \\
\hline
\end{tabular}

Note. M: mean; SD: standard deviation.

The same pattern of increase is discernable in Fig.1. Gains in vocabulary knowledge are most notable in receptive knowledge of meaning and form (RMF). While productive knowledge of associations follows the same trend, it is much less in quantity.

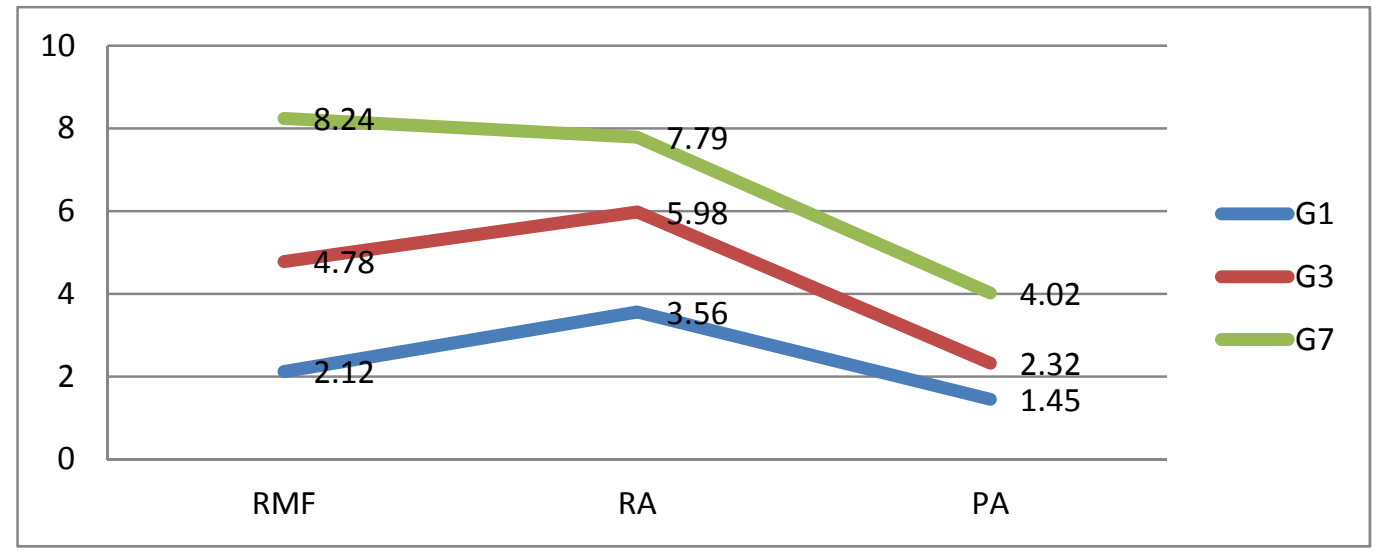

Figure 4. Mean scores for each group on vocabulary test.

To discern significant differences among the three experimental groups, ANOVA and its post hoc Tukey (or Kruskal-Wallis and post hoc LSD) were used. As shown in Table 5, the mean score differences for receptive knowledge of meaning and form and receptive knowledge of associations reached statistical significance for all groups of participants. That is to say, increasing exposure frequency from one to three and from three to seven encounters had a significant effect on the extent the participants developed their vocabulary knowledge. For productive knowledge of associations, however, the mean score differences were not significant when one to three encounters or three to seven encounters were compared. It seems for this aspect of lexical knowledge, at least seven encounters to the TWs were required for significant gains to appear.

Table 5. Group comparisons on vocabulary test

\begin{tabular}{|l|c|c|c|}
\hline \multicolumn{1}{|c|}{ Sub-test/Group } & G1 / G3 & G3 / G7 & G1 / G7 \\
\hline Receptive Knowledge of Meaning and Form & $0.039^{*}$ & $0.030^{*}$ & $0.000^{*}$ \\
Receptive Knowledge of Associations & $0.035^{*}$ & $0.031^{*}$ & $0.003^{*}$ \\
Productive Knowledge of Associations & 0.056 & 0.067 & $0.000^{*}$ \\
\hline
\end{tabular}

Note. $*=p<.05$ 


\section{Discussion}

Of primary importance to the present study was to explore the possible effects of implicit exposure on the acquisition and development of vocabulary knowledge. In other words, the study sought to answer whether or not increasing written input would result in a better outcome in terms of lexical knowledge. In this regard, the findings confirmed that exposure frequency had a positive effect on vocabulary knowledge as a continuum of one to seven encounters could show. This is in line with previous study which had reported the effectiveness of exposure frequency (e.g. Chen \& Truscott, 2010; Web, 2007).

The researcher was also interested to know if the effects of exposure frequency varied across different aspects of vocabulary knowledge or not. As mentioned above, three aspects of lexical knowledge that is receptive knowledge of meaning and form (RMF), receptive knowledge of associations (RA) and productive knowledge of associations (PA) were taken into account. Group comparisons on these subknowledges through a scale of one-three-seven encounter revealed that receptive knowledge of meaning and form (RMF) as well as receptive knowledge of associations (RA) responded faster to implicit exposure frequency than productive knowledge of associations (PA). While gains in vocabulary knowledge for RMF and RA were significant even after three encounters, for this latter subknowledge, at least seven encounters were needed to reach significant results. Web (2007) also found smaller gains in semantic areas of vocabulary knowledge than orthography. Moreover, as he indicates receptive measures are more sensitive to small gains of knowledge.

In addition, since culturally-loaded words have multiple associations with other words and have a wider grid of semantic features, it is plausible to assume that such words require more exposure to be fully acquired. This, in turn, justifies why in productive knowledge of associations more encounters to the TWs were required. As for receptive knowledge of meaning and form (RMF) and receptive knowledge of associations (RA), however, the participants' lexical knowledge was measured at recognition level (not production). Hence, it might be the case that even less exposure frequency could suffice.

\section{Conclusion}

The present study embarked on investigating an important area of L2 vocabulary acquisition and implicit learning. That is, whether or not increasing the amount of exposure to L2 words while reading could affect the acquisition and development of lexical knowledge. Moreover, it was of particular importance to discern which aspects of this knowledge were more involved. The findings in general confirmed the effectiveness of such treatment. Moreover, it seems productive knowledge of associations needed more exposure for significant gains.

\subsection{Implications}

This study suggests that implicit exposure to L2 vocabulary could help learners develop different aspects of vocabulary knowledge. In this regard, 'extensive' reading that is reading large amounts of reading materials within a longitudinal program can be helpful (Day \& Bamford, 2002). It is recommended that EFL learners be encouraged to read authentic texts. For those elementary or intermediate learners, however, such texts may not be feasible 
because the vocabulary load is usually too high for them (Pellicer-Sanchez \& Schmitt, 2010). Therefore, as the second option, graded readers are recommended which are easily adaptable to learners' proficiency level (Al-Homoud, 2007).

In addition, language teachers are recommended to incorporate culture (as a core component) into their teaching plans. Learning tasks, for instance, may spin around cultural topics. Combing implicit and explicit approaches also would be helpful to handle culturally-loaded words. Cultural portfolios are also effective teaching tools to combine learning a second (or foreign) language with cultural notions. Using these portfolios, teachers can encourage learners to collect and categorize the artifacts they come across over time. In addition, it helps them become aware and reflect on language as a part of culture and culture as part of language.

\subsection{Limitations of the Study}

As any piece of research, this study was also limited in a number of ways. Firstly, the participants were all adult learners at intermediate level of language proficiency at two Iranian universities. Therefore, the sample of the participants could be larger and more representative. Secondly, only quantitative measures of vocabulary knowledge were used. This might have affected the validity of the findings. Finally, retention was not taken into account. Thus, no conclusion can be drawn on how long the observed effects could last.

\subsection{Suggestions for Future Studies}

It is recommended that interested researchers follow the same line of research with a more representative sample of participants (including various age groups and proficiency levels). Moreover, longitudinal studies are favorable to see to what extent any observed gains in vocabulary knowledge remain at later periods of time. In the end, using a mixed-methods research design would be helpful in obtaining more reliable results.

\section{References}

Al-Homoud, F. (2007).Vocabulary Learning via Extensive Input. Unpublished doctoral dissertation, University of Nottingham, UK.

Brent, M.R., \& Siskind, J.M. (2001).The role of exposure to isolated words in early vocabulary development. Cognition, 81, B33-B44. http://dx.doi.org/10.1016/S0010-0277(01)00122-6

Broady, E. (2008).Fragmentation and consolidation: recent articles on vocabulary acquisition. The Language Learning Journal, 36(2), 259-265. http://dx.doi.org/10.1080/09571730802390833

Brown, D. (2011). What aspects of vocabulary knowledge do textbooks give attention to? Language Teaching Research, 15(1), 83-97. http://dx.doi.org/10.1177/1362168810383345

Brown, R., Waring, R., \& Donkaewbua, S. (2008). Incidental vocabulary acquisition from reading, reading-while-listening, and listening to stories. Reading in a Foreign Language, 20(2), 136-163. 
Chen, C., \& Truscott, J. (2010).The effects of repetition and L1 lexicalization on incidental vocabulary acquisition. Applied Linguistics, 31(5), 693-713. http://dx.doi.org/10.1093/applin/amq031

Day, R. R., \& Bamford, J. (2002).Top ten principles for teaching extensive reading. Reading in a Foreign Language, 14(2).Retrieved from http://nflrc.hawaii.edu/rfl/October2002/

Eckerth, J., \& Tavakoli, P. (2012).The effects of word exposure frequency and elaboration of word processing on incidental L2 vocabulary acquisition through reading. Language Teaching Research, 16(2), 227-252. http://dx.doi.org/10.1177/1362168811431377

Heidari-Shahreza, M. A., \& Tavakoli, M. (2012).The effects of repetition and L1 lexicalization on incidental vocabulary acquisition by Iranian EFL Learners. The Language Learning Journal, 40, 1-16. http://dx.doi.org/10.1080/09571736.2012.708051

Heidari-Shahreza, M. A., Moinzadeh, A., \& Barati, H. (2014a).The effect of exposure frequency on incidental vocabulary acquisition. GEMA Online Journal of Language Studies, 14(1), 43-55.

Heidari-Shahreza, M. A., Moinzadeh, A., \& Barati, H. (2014b).A comparative investigation into effects of L1 lexicalization and cultural loadedness on incidental vocabulary acquisition and retention. International Journal of Research Studies in Language Learning, 3(5), 83-96. http://dx.doi.org/10.5861/ijrs11.2014.698

Hellman, A.B. (2011). Vocabulary size and depth of word knowledge in adult-onset second language acquisition. International Journal of Applied Linguistics, 21(2), 162-182. http://dx.doi.org/10.1111/j.1473-4192.2010.00265.x

Horst, M., Cobb, T., \& Meara, P. (1998). Beyond A Clockwork Orange: acquiring second language vocabulary through reading. Reading in a Foreign Language, 11, 207-223.

Hulstijn, J., Hollander, M., \& Greidanus, T. (1996). Incidental vocabulary learning by advanced foreign language students: the influence of marginal glosses, dictionary use, and reoccurrence of unknown words. The Modern Language Journal, 80, 327-339. http://dx.doi.org/10.1111/j.1540-4781.1996.tb01614.x

Kim, Y. J. (2011). The role of task-induced involvement and learner proficiency in L2 vocabulary acquisition. Language Learning, 61(1), 100-140. http://dx.doi.org/10.1111/j.1467-9922.2011.00644.x

Kirn, E., \& Hartmann, P. (2002).Interactions 1: Reading. New York: McGraw-Hill/Contemporary.

Lado, R. (1972). Patterns of difficulty in vocabulary. In H. B. Allen \& R. N. Campbell (Eds.), Teaching English As a Second Language. New York: McGraw-Hill.

Liu, D., \& Zhong, S. (1999). Acquisition of culturally loaded words in EFL. Foreign Language Annals, 32(2), 177-187. http://dx.doi.org/10.1111/j.1944-9720.1999.tb02390.x

Meara, P. (2012). The bibliometrics of vocabulary acquisition: An exploratory study. RELC 
Journal, 43(1), 7-22. http://dx.doi.org/10.1177/0033688212439339

Nation, I. S. P. (1990).Teaching and Learning Vocabulary. New York, NY: Heinle and Heinle Publishers.

Nation, I. S. P. (2001).Learning Vocabulary in another Language. Cambridge: Cambridge University Press. http://dx.doi.org/10.1017/CBO9781139524759

Nation, I. S. P. (2013).Vocabulary acquisition in second language acquisition. In C. A. Chapelle (Ed.), The Encyclopedia of Applied Linguistics. Oxford: Blackwell Publishing Ltd.

Pellicer-Sanchez, A., \& Schmitt, N. (2012).Scoring Yes-No vocabulary tests: Reaction time vs. nonword approaches. Language Testing, 29(4), 489-509. http://dx.doi.org/10.1177/0265532212438053

Pigada, M., \& Schmitt, N. (2006). Vocabulary acquisition from extensive reading: A case study. Reading in a Foreign Language, 18(1), 1-27.

Qian, D.D., \& Schedl, M. (2004).Evaluation of an in-depth vocabulary knowledge measure for assessing reading performance. Language Testing, 21(1), 28-52. http://dx.doi.org/10.1191/0265532204lt273oa

Rott, S. (1999).The effect of exposure frequency on intermediate language learners' incidental vocabulary acquisition and retention through reading. Studies in Second Language Acquisition, 21, 589-619. http://dx.doi.org/10.1017/S0272263199004039

Rott, S. (2007).The effect of frequency of input-enhancements on word learning and text comprehension. Language Learning, 57(2), 165-199. http://dx.doi.org/10.1111/j.1467-9922.2007.00406.x

Saragi, T., Nation, P.,\& Meister, G.F. (1978).Vocabulary learning and reading. System, 6, 72-78. http://dx.doi.org/10.1016/0346-251X(78)90027-1

Waring, R., \& Takaki, M. (2003). At what rate do learners learn and retain new vocabulary from reading a graded reader? Reading in a Foreign Language, 15, 130-163.

Webb, S. (2007). The effects of repetition on vocabulary knowledge. Applied Linguistics, 28(1), 46-65. http://dx.doi.org/10.1093/applin/aml048

Webb, S. (2013). Depth of vocabulary knowledge. In C. A. Chapelle (Ed.), The Encyclopedia of Applied Linguistics. Oxford: Blackwell Publishing Ltd. 


\section{Macrothink}

\section{Appendix}

Appendix 1. Selected TWs

\begin{tabular}{|l|l|l|}
\hline \multicolumn{1}{|c|}{ Verb } & \multicolumn{1}{c|}{ Noun } & \multicolumn{1}{c|}{ Adjective } \\
\hline Gamble & funeral & abstinent \\
\hline fast & girlfriend & drunk \\
\hline date & pet & moral \\
\hline veil & undertaker & religious \\
\hline mourn & virgin & \\
\hline greet & dowry & \\
\hline marry & charity & \\
\hline pray & pilgrimage & \\
\hline
\end{tabular}

Appendix 2. Arrangement of the Texts

\begin{tabular}{|c|ccccccc|c|}
\hline Group & \multicolumn{7}{|c|}{ Arrangement of Main and Distracter passages } & Frequency \\
\hline G1 & $\mathrm{D}_{1}$ & $\mathrm{D}_{2}$ & $\mathrm{D}_{3}$ & $\mathrm{D}_{4}$ & $\mathrm{D}_{5}$ & $\mathrm{D}_{6}$ & $\mathrm{M}_{7}$ & $\mathbf{1}$ \\
\hline G3 & $\mathrm{M}_{1}$ & $\mathrm{D}_{2}$ & $\mathrm{D}_{3}$ & $\mathrm{M}_{4}$ & $\mathrm{D}_{5}$ & $\mathrm{D}_{6}$ & $\mathrm{M}_{7}$ & $\mathbf{3}$ \\
\hline G7 & $\mathrm{M}_{1}$ & $\mathrm{M}_{2}$ & $\mathrm{M}_{3}$ & $\mathrm{M}_{4}$ & $\mathrm{M}_{5}$ & $\mathrm{M}_{6}$ & $\mathrm{M}_{7}$ & $\mathbf{7}$ \\
\hline
\end{tabular}

\section{Copyright Disclaimer}

Copyright for this article is retained by the author(s), with first publication rights granted to the journal.

This is an open-access article distributed under the terms and conditions of the Creative Commons Attribution license (http://creativecommons.org/licenses/by/3.0/). 